\title{
Intelligent Process Automation of Industries Using Artificial Intelligence and Machine Learning
}

\author{
${ }^{1}$ Filippo Fabrocini and ${ }^{2} J i a n ' a n$ Sun \\ 1,2 School of Software, Beijing Institute of Technology, Beijing, China, 100081. \\ 1'rocinifilippo@bit.edu.cn
}

\author{
Article Info \\ Journal of Computing and Natural Science (http://anapub.co.ke/journals/jens/jens.html) \\ Doi: https://doi.org/10.53759/181X/JCNS202101009 \\ Received 10 November 2020; Revised form 12 January 2021; Accepted 20 January 2021. \\ Available online 05 April 2021. \\ (C)2021 Published by AnaPub Publications.
}

\begin{abstract}
The application of Disruptive Technologies (DT), using Artificial Intelligence (AI) and Machine Learning (ML), is still a challenge for many industries in the modern age. Quick transformation of business' models and enhancement of consumer expectation are fundamental elements of Intelligent Process Automation (IPA) to boosting delivery and production of goods and services. In this research contribution, we will evaluate IPAs and their influence in the management of industries. In that case, major elements of AI and ML will be discussed comprehensively. Areas of application and analysis will be discussed in relation to digital industries. The results in this contribution will be used as a recommend action plan for industries to enhance their management and optimization when it comes to AI and ML.
\end{abstract}

Keywords - Intelligent Process Automation (IPA), Artificial Intelligence (AI), Machine Learning (ML)

\section{INTRODUCTION}

Due to increased digitalization, Disruptive Technologies (DT) has gained popularity among many modern industries [1]. In that case, there has been increased attention on elements of Machine Learning (ML) and Artificial Intelligence (AI) in the digitalization of industries in the modern age. ML is closely applied with AI as a process used to automate the process of detecting data patterns. The identification of these patterns is done using ML algorithms that are certainly tailored to handle big and complex data. ML and AI have a significant application to modern industrial environment.

AI-powered maintainability robots in anomaly detection are one of the applications of AI and ML in industrial digitalization [2]. Most industrial production ecosystems necessitate periodic maintainability of industrial machines. This application has become especially fundamental with the enhancement of awareness concerning the safety of workplaces and climatic changes. Nonetheless, in most cases, fundamental elements such as installation are challenging to maintain and reach since they require considerable efforts from service experts and engineers, and drastically advancing costs associated with maintainability and production. In the future, the works of various service experts and engineers could completely be automated with the application of AI-powered robots that are capable of inspecting, maintaining and repairing production installations. These intelligent robotic systems have the capacity to navigate to the essential location and undertake particular tasks that could have been done by humans. Resultantly, this permits production facilities to minimize the available workload for humans, enhance operation safety and save on budget without reducing the reliability aspect of the industries.

Moreover, IPA and installations would be equipped with intelligent sensors that are capable of gathering essential data on production volumes, temperature and mechanical vibrations. ML algorithms with the aim of identifying and evaluating production anomalies can evaluate these sets of data. For instance, identifying the changes in production volumes might indicate a leak within a specific plant, a transition in mechanical vibration pattern of machines indicating parts that require replacement and vibrations in temperature datasets, which suggest that the industry has to be calibrated.

Digital twin technologies to assist model-centred system engineering are another application of AI and ML in industrial digitalization [3]. Stringent safety guidelines and higher efficacy demands are resulting in an advanced engineering ecosystem, hence making the application of Computer-Assisted Engineering (CAE) models challenging. In that case, novel innovations e.g. Digital twins, ML and AI are becoming significantly essential to model-centred system engineering and in the operation of machines such as gas compressors, mechanical drives and gas turbines. Nonetheless, by connecting digital twin with the sensory output information from the physical ecosystem, normally captured by Internet of Things (IoT) data, the technology permits actual-time monitoring of machine cases in a vibrant manner.

The connection allows the industrial manufacturers to run simulations on the digital twins, with respect to data retrieved from physical systems, which are operational. In this manner, digital twins can be given with the particular 
information during simulations: individual maintainability history and services; operational histories and ecosystem data retrieved from IoT devices; product design histories and machine configuration during operations. The results of simulations can be compared to real-life outcomes, out of the operational physical systems, which can be evaluated using ML models along with CAE. ML models are utilized in the provision of valuable insight concerning physical twins, which makes it possible to design maintainability requirements and design improvements within particular production ecosystems.

Recognition of speech using recurrent neural networks is another application of AI and ML in IPA. One of the critical technologies provided by $\mathrm{AI}$ and $\mathrm{ML}$ is speech recognition [4]. This provides the capacity of programs and machines to accept, process and evaluate spoken sentences, hence developing interactions between machines and humans. Speech recognition has wide-range application within the business ecosystem.

The first category of speech recognition application concentrates on the reduction of task costs, which are being accomplished by human attendants e.g. automation of tasks in operating services. Earlier on, operating service calls were dealt with human workers; as such, the application of voice recognition software has allowed partial or full automation of the entire process. Incoming calls are dealt with systems of speech recognition, which identifies the problem of the caller by requesting for a series of questions. Based on the feedback, callers are redirected to human attendants specialized in matters concerning the caller's issues. This has allowed operating services to enhance in efficiency and provide effective customer services and experiences using limited resources.

Critical speech recognition software can undertake detailed data mining on wide-range audio files that have been retrieved from customer calls. These data analyses might provide fundamental demographic data concerning the caller i.e. sentiment, emotion, accents, age and gender. This data permits the business to gain critical insight concerning the customer base, launching targeted marketing campaign, enhancing support and boosting the performance of sales.

The second category of speech recognition application within the context of IPA is automatic transcription software for texts. This software permits the transition of video and audio fragments into text documents that integrate spoken texts from imported files. It might be fundamental to acquire transcription on board meeting, stakeholder meeting and conference calls to effective transfer data to individuals that have not attended the meeting.

The application of data lakes for AI is another key application essential in IPA. The increased adoption of disruptive technology is various aspects of the human life has amounted to an enhanced connectivity between businesses, customers and persons and has therefore brought an increase in information transfer between various devices. With the every-increasing capacity of storage and evolving data collection methods, this has amounted to big data becoming readily accessible by industries. Nonetheless, industries that enter the landscape of big data have to seek solution to efficiently manage such big data. This is typically achieved by data lakes: models that permit the storage of unstructured and raw data concerning the industry and its customers to be used in the future. Data lakes need not to be mistaken with data warehouses i.e. Datamarts, which are typically utilize systems storing structured information that have to be analysed or for answering simple queries.

The significance of applying data lakes is that all information entering the industry will be stored for future use. It normally happens that information points might be vital at a particular point; but would provide value to the industry within the near future. In this manner, data lakes provide a means of storing data hence making it readily available in its initial format. Storing such records of data might be costly and might necessitate larger parts of data storage capacities to be available. In that case, it is recommended that industries first quantify its costs and merits of applying data lakes before following a particular action plan.

AI for Information Technology (IT) operations is also another application in the automation of industries [5]. Technology experts are experiencing first-hand how the advancement of data is changing their jobs hence necessitating them to utilize novel tools in monitoring data, identifying management problems and recommend potential solutions. Nonetheless, it has turned out to be challenging to manage large streams of data in an effective manner, hence leading to potential delays when essential issues require identification and resolving. In that case, technologies such as ML and AI have turned out be fundamental in assisting IT experts in managing and resolving ITrelated operation issues. This has amounted to an entirely novel field, typically known as AIOps, where AI is applied in the management of IT operations. The platforms of AIOps are capable to partially and fully replace particular parts of IT operations eg monitoring performances, evaluate IT-based events and performing IT service management by applying big data analytics and ML strategies. In this manner, they relieve the available workloads of IT personnel hence permitting them to concentrate on IT activities, which provide more strategic value to industries.

ML for customer marketing and intelligence is another key application for the automation of industries. ML algorithms have proven to the fundamental in rapidly evaluating large counts of data points and provide insights, which are comprised within datasets. In that case, ML algorithms can be utilized with the aim of gaining valuable insight regarding the behaviour of consumers and creating more effective marketing approaches. One ML approach, which has proven to be relevant for such purposes are the recommender models. The recommender models, according to its name, are capable to determining the kind of products a particular user would need, provided the data for users' past purchases.

Recommender models are regularly utilized by large companies such as Amazon, Youtube, and Netflix to provide its customers with recommendation of products, which are aligned with needs and interests. Applying such recommender models has some advantages to both the industry and its consumers. First, consumers are targeted with the industry's products, which are aligned to particular needs; hence increasing their satisfaction level and customer experience. Next, industries are capable of providing effective consumer support and simultaneously advancing their sales level because consumers are likely to purchase additional products within the intent to do so. 
In this section, an introduction of industrial revolution based on the implementation of ML and AI has been done. The present industrial revolution from the application of mechanical production over the assembly line work and division of labour to the application of automatic and electronic production are considering in modern-day industries. We arrived at 4.0 industrial revolution i.e. digitalization where the concepts of IoT, Intelligent Manufacturing, Big Data, Machine-to-Machine Communication and Cloud Computing are considered. The main purpose of this contribution is to evaluate the application of $\mathrm{AI}$ and $\mathrm{ML}$ in the field of industrial management and automation. The results in this contribution will show the impact, advantages and disadvantages of ML and AI for process optimization and process steering. Section II below presents definition and analysis of terms fundamental for this research. The remaining part of the paper is arranged as follows: Section II presents the definition of terms. Section III is the literature review section. Section IV is the methodology section. Section V presents the results of the research. Section VI is the discussion part. Lastly, Section VI concludes the paper.

\section{DEFINITION OF TERMS}

Intelligent Process Automation (IPA)

The terminology Intelligent Process Automation (IPA) has existed since the early 1960s with wider availability and application in mainframe computer. Considering further advancement steps in digitalization e.g. in 1980, the introduction of Business Intelligence (BI) and CRM tools to link objects, 3D printing, AI and block chain technologies, the advent of IPA and digital technologies transformed the approach of collaboration execution at different levels of the economy in a radical manner. In that case, IPA implies to the speedy change in cooperation because of the implementation of digital initiatives and innovation and the incorporation of the various aspects of the human life and the community at different dimensions.

With the advent of novel digital initiatives, such as; social networks, big data and mobile technologies, industries are undertaking multiple approaches to exploit and explore the benefits. The typically incorporate the transformation of key industrial operations and effects of industrial products and process, including organizational structures, as industries have to establish management practices to effectively manage complex transformations. Therefore, the community in general is facing radical and fast transition due to the maturity of digital technologies and ubiquitous penetration of the business market. To integrate the advancing demand of clients, industries are facing critical competition because of globalization hence facilitating the need to digitalize, focusing on sustainability and achieving competitive advantage.

'Digital pioneers' e.g. Google, Facebook, and Amazon, have developed into powerful behemoths whereas industries that dominated their businesses found their traditional proposition of value to be under threat. Nonetheless, irrespective of multiplicity of technological recipes and novelties for the implementation, whether in the industry, private life, and governance, IPA is facing challenges than expected. It is unfortunate that there are various recent examples of organizations, which have not be able to maintain the pace of novel digital realities i.e. bankruptcy of movie rental, significantly amounting from the company's incapacity to develop and implement novel digital-based business models. Effective IPA necessitates industries to formulate wide-range capacities that will vary in significance depending on the context of business and particular needs of the industry. Digital technologies have to be centralized to how the industry operates and the industry should rethink and reinvent their organizational models to remain competitive. To this extent, the subsection below provides a definition of business process management.

\section{Business Process Management}

Business process management is a concept of management that is used to control, adapt and optimize industrial processes. A detailed definition for business process management from the European Association of Business Process Management (EABPM) is that the concept is considered a systematic approach that steers, monitors, measures, documents, executes shapes and captures both automated and non-automated processes to reach sustainable and coordinated company targets. The main purpose of business process management is to enhance corporate performance through the optimization and management of business processes in an industry.

Business process management can be defined as corporate organizational process management and optimization over the same system e.g. SCM, CRM and ERP and the complete integrated system. The allocation of business process management and system signifies the execution and coordination of processes step-by-step. Through evaluating, identification and monitoring of process troubles and issues, business process management of systems depicts where processes had issues and allows businesses to optimize process issues.

Artificial Intelligence (AI)

Two approaches define AI. The first one draws humans as comparisons and the second one AI is considered by a list of various work areas and applications. For instance, AI integrates 'the development and theory of computer systems capable of performing tasks normally necessitating human intelligence.' AI evaluates the manner in which intelligent behaviors can be captured and understood using computers and how problems can be resolved using computers requiring interoperability. AI integrates the grouping of computer science that handles the assessment of various issues e.g. flow text, speech recognition and robotics, images and video process.

Based on the scope of this research, AI can be defined as a segment of informatics that is applicable to computer science to effectively pattern proceedings of humans in problem solving and communicate them to computers to invent effective and novel solutions and course of actions. In that case, AI signifies a computer program that runs on devices or information centers with the competency to interact with the ecosystem. The definitions of AI are short is 
such a way that the terminologies "intelligent human behaviors" and "intelligence" are not well understood and defined yet.

In 1950, in an analysis about computing intelligence and machinery, a British mathematician, aspect a question on whether a program can be considered to be intelligent or not. In his study, the test person can communicate through computer terminals with two partners that are invisible i.e. human beings or program. Finishing, the test individual has to decide which is which. Two forms of AI are differentiated in literature as show in Fig 1 below. It is fundamental to comprehend the differentiation AI for this research.

\section{Strong AI}

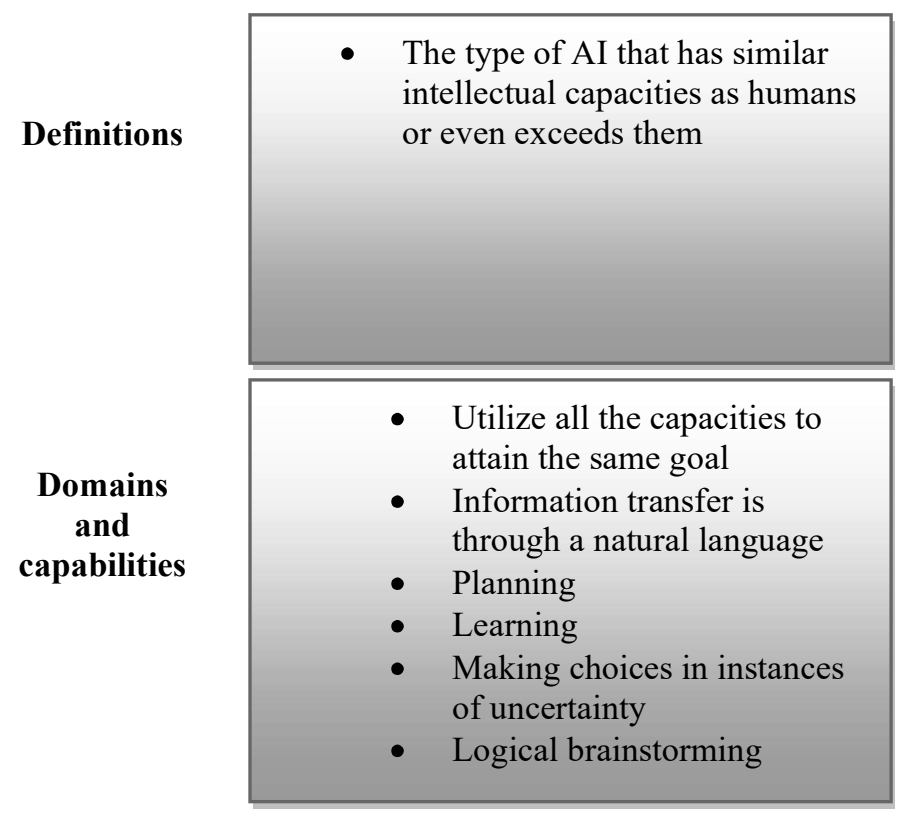

Weak AI

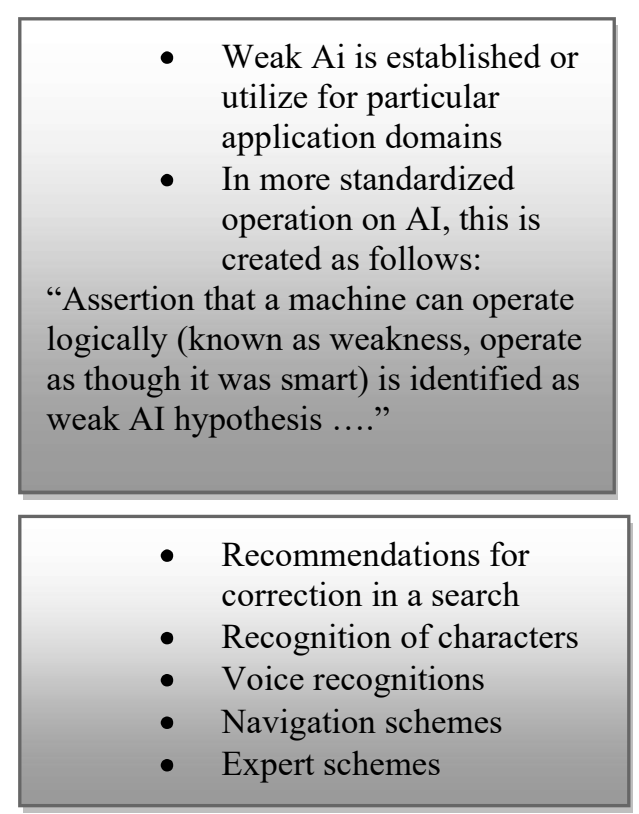

Fig 1: Differentiation between weak and strong AI

Presently, AI can be identified in various forms of weak AI in the human ecosystem. Examples of these include object-based programming languages, translation models and graphical users as well as smart games e.g. chess can be installed in computers. The programs of chess have recently surpassed the intelligence of the best player e.g. IBM deep blue super-computers. More samples of AI include control systems and voice recognition e.g. intelligent agents and bots, echo, Siri and Cortana. These can potentially interact independently during phone conversations. The most recent success in $\mathrm{AI}$ is stimulated by ML.

Defining AI can be referenced from Marvin Lee Minsky who defined it as a science of creating machines to do something that would require intelligence. It necessitates high-level mental procedures e.g. critical thinking, memory, and perceptual learning. AI is a science of developing computer programs, which focus on completing tasks that necessitate intelligence if they are completed by humans. In that case, no human activities seem to be out of reach; shifting from one point to another, studying reasoning, and creativity and socializing. Nonetheless, we are still behind in creating machines, which are capable of outperforming human intelligence in all segments. Based on logic, researchers figure out $\mathrm{AI}$ as a standard measure of intelligence that is more than human capacities. The approach to AI utilizes tools provided by the logic of mathematics to formalize tasks that are more complex by AI machines. The main problem here is that this approach has to handle the formalization of tasks.

Machine Learning (ML)

Machine Learning (ML) is defined as a branch of AI, which describes mathematical approaches that allow a system or machines to independently generate knowledge out of experience. ML describes the creation of algorithms with respect to empirical data, including training data focusing on the improvements of predictions and result optimization because of learning processes. The terminology algorithm can be defined as critical sequence of actions meant to solve a specific task. The establishment of ML is because of the research of computer learning theory and recognition pattern. Algorithmic application of ML happens with the unsupervised and supervised learning as shown in Fig 2 below. 
Supervised learning

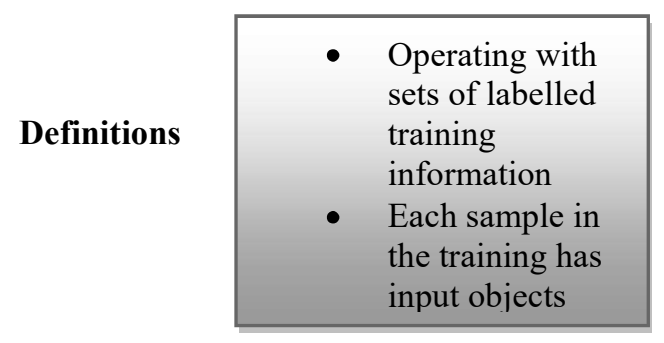

Unsupervised learning

Fig 2: Supervised and unsupervised ML

\section{LITERATURE REVIEW}

K. Tangrand and B. Bremdal in [6] have presented the theories showing that methods of Deep Learning (DL) with Neural Networks (NN) are always considered when AI is mentioned. The rapid enhanced progress of DL over the decades can significantly lead to powerful hardware used in computing activities. In addition, the increase in data amounts, which is fundamental for initial training of $\mathrm{NN}$ have a significant impact. After initial training, DL of procedures incorporates the aspect of learning during the operating application that increases recognition results and accuracy. This can be deduced that close to the algorithms, big data is critical in DL and therefore useful in AI.

O. Prokopenko and V. Omelyanenko in [7] have evaluated the aspect of innovation in action. In the study, two narratives were presented to evaluate how industries consider using PaaS to thrive in the competitive market. The first narrative is where the industrial LLC provides clients with a 'leg up'. Industries, a novel dimension LLC, are considered digitally powered start-ups, which provide networking marketing systems that are particularly for one of the world's largest B2B segment. The industrial segment is constantly disrupting its industrial norms with custom software, which can be accessed by its auto dealers through PaaS mobile cloud services.

S. Kwak in [8] argues that B2C auto sales have significantly evolved due to online technology; however, motor auctions and trades are still considered in their traditional modes. In a classical framework, motors are auctioned and spotlighted for a few minutes and their attention can be shifted to another motor. Industries are revolutionizing using mobile apps (based on mobile cloud solutions, which are a part of PaaS). The advancement makes the auto-trading sector to be a round-the-clock experience compared to industries that are event centric. The industry's mobile apps and web-centered and appraisal tools provide online market places from auto trading and actual-time reporting to track pre-owned inventory data and track appraisal statistics.

I. Lee and Y. Shin in [9] evaluated ML as a driving force that stimulates changes at three levels: business models, business processes, and occupations/tasks. One of the examples of occupation/task redesign is the application of the machine vision system that identifies cancer cells, in freeing up radiologists to concentrate on critical cancer cases, to coordinate activities with other physicians and to communicate with patients. One of the examples of process redesigning includes the reinvention of workflows and layouts of industrial fulfillment centers after the introduction of optimization algorithms and robots using ML.

H. Yang in [10]evaluated business models and concluded that they have to be rethought to apply ML in industries. An effective model, for the case of the movie and music industry, can provide personalized subscriptions to custom stations, which played and predicted music a specific customer would with to hear. It should be noted that ML models hardly integrate the complete business model, job or process. In most cases, they complement human behaviors and actions that can make work to be more valuable. The most effective protocol for novel division of labor is rarely, if ever, 'provide tasks to machines.' Instead, in case successful completion of the procedure necessitates 10 steps, one of them might be automated whereas others are considered more valuable to humans.

For example, chat room sales and support systems at Udacity did not try to develop bots, which could handle all the conversations; instead, it advised sale persons on how they can enhance performance. Humans remain in charge to enhancing efficiency and effectiveness. The strategy is typically more feasible compared to the designing of machines, which could handle all the activities for humans. This typically amounts to satisfying work for individuals involved and enhances the results of customers. Implementing and designing novel combinations of technologies, capital assets and human needs to meet the demands of clients, high-degree planning and creativity is needed. Machines cannot do this task better. In that case, entrepreneurs and business managers are essential in the age of ML.

P. Addo, D. Guegan and B. Hassani in [11] assessed the limits and risks of ML. From the study, the researcher evaluates the second wave of ML models and commented that is has low interpretability. This shows that humans face issues in figuring out how models and systems arrive at decisions. Deep Neural Networks (DNN) may be considered to have hundreds of connection, and each of them contributes a minor amount of decision. Resultantly, the systems projections tend to resist clear and simple explanations. Unlike human beings, machines are not good at providing explanations. Machines cannot entirely be relied to provide rationales as to why a particular individual was rejected or accepted for a task or why a certain medicine was recommended. 
M. Leo, S. Sharma and K. Maddulety in [12] argues that machines know more than what they tell. This presents three critical risks. The first one is that machines might have a hidden bias, which might not be derived from the designer's intent; but from the data given to train these systems. For example, in case a system learns the kind of job applicant to approach for an interview by using different sets of data regarding to choices made by past human recruiters, it might inadvertently master how to perpetuate their biases, ethnicity, gender and race. In addition, the biases might not appear as an explicit protocol; but rather, embedded in more subtle interaction among wide-range factors considered.

G. Keilbar and W. Wang in [13] evaluated another risk that neural network system bears compared to traditional systems. It is considered that neural networks bear statistical truths instead of literal truths. This can therefore make it challenging, if not impossible, to enhance with full certainty that models will operate in all cases, mostly in situations that were not represented in training information. The deficiency of verifiability can be a major issue in missioncritical ML applications e.g. in the control of nuclear energy plants, or when making life-threatening decisions.

$\mathrm{S}$. PK in [14] argues that when systems of ML make errors, it can be challenging to correct or diagnose what is exactly wrong within the system. The underlying system structure, which stimulated the solution, might be unimaginably challenging and the remedy might be far from its optimal in case the condition under which the ML system was trained changes. Whereas the various risks are considered real, effective benchmarks are not perfect; but considered the most effective alternative. Overall, humans also have biases and make mistakes when it comes to making proper decisions. Irrespective of the risks evident in the application of ML and AI when it comes to IPA of industries, ML system can be used to enhance industrial activities.

T. Wuest, D. Weimer, C. Irgens and K. Thoben in [15] present the advantages of ML system in enhancing business activities. Although these systems have risks, they can be advanced as time goes to provide consistent remedies when provided with the same sets of data. S. Konopatov and N. Saliyenko in [16] argue that modern platform-centered business models are transforming the present rules of business competition. Sharp competitive edges emerge whenever industries utilize PaaS to link members, customers, workers, information and data within their value chain. These platforms deploy digitalized and automated processes, which reach wide-range business divisions.

P. Pereira in [17] argued that the advantages of using ML system are achieved by applying PaaS to reach into new channels and discern novel opportunities to create value to consumers. Conversational chatbots and AI, for instance, the industry using PaaS might not be rooted in a continuous loop of learning. Every PaaS element can feed data back in so that it can be linked to constantly advancing pool of data hence aiding in the advancement of AI based on every iteration. The major concept is that cloud technology operates autonomously. AI and ML do fundamental but mundane tasks, whereas human resources are integrated to enhance innovation on behalf of clients and to uncover novel opportunities of monetizing. Using this approach, the industrial can accelerate innovations and leave competitors wondering how they even missed the turn of clients' preference.

A. Rogers, O. Kovaleva, M. Downey and A. Rumshisky in [18] presented an assumption that AI deals with getting machines to execute tasks that require intelligent behaviors. Some of the examples include verbalizing plans, playing games, making conjectures, comprehending human speech and using apparatus. AI concentrates on how to get computers and machines perform similar tasks, though not necessarily the same manner that animals or humans do. V. Reshetnyk in [19] evaluated the term automation and commented its invention in the automobile industry in the late 1940s to illustrate the application of automatic controls and devices in mechanical production line. The introduction of the term is credited to D.S. Tougher at the Passage Motor Industry at that time.

S. Dey and A. Das in [20] present the need for automations in industries. It is argued that automations are required in different sectors of the human life, from workshop line robot, automated doors to marketable procedural automations. Presently, AI is a prevailing concern among industries in enhancing automated processes. For instance, we presently have chatbots completing consumer services. AI is not only applied in industries, but in smart homes as well.

\section{METHODOLOGY}

For the proposed analysis and evaluation of using AI and ML, an analysis with interview features and a shadowing with digital industries were executed to assess the status quo at industries regarding this topic. With reference to the facts that have been analyzed and AI strategy, a recommendation are considered to survey participants and industries for automated optimization of processes using AI and ML.

In this study, 25 digital U.K. companies were survey. A digital company is featured by its preponderant application of data technology for undertaking primary business activities in a value added aspect. Approximately, $75 \%$ of the survey questions were about the application of data technologies and business activities. About $25 \%$ of the survey questions were about the possibility for evaluating daily tasks by shadowing the process management group. Shadowing denotes to the attendance of members at daily business to consider working. For the research, an anonymous method of survey was utilized.

It was considered that the subject present their expectations, experience, and knowledge and there real execution of process management. The people being target in the research included Chief Executive Officers (CEOs), Chief Information Officers (CIOs), IT process managers, and Chief Technology Officers (CTOs). The baseline of the research is considered 65 valid replies of surveys replied from an overall of 75 surveys that were requested. The survey integrated 5 open questions and 30 closes. The survey, through interview proceedings, considered about 35 to 
45 minutes for every individual. Close to the survey, shadowing was considered at the chosen firms. As observers included the process flows, processes themselves and process management tasks, as well as essential applications analyzed and watched. The time of shadowing is different within industries i.e. between 4-8 hours. After that, detailed comparison and evaluation of collected data happens to identify the baseline and deviations. Based on shadowing, an evaluation of theoretical execution and appreciation was considered.

\section{RESULTS}

The findings of the survey are presented and the recommendations drawn for applying AI and ML in this sections. Approximately $87 \%$ of the involved managers of the twenty-five companies accepted the survey and interviews, which integrates shadowing to evaluate the baseline of executing automated business process management in their companies. The surveys indicate that each industry and $100 \%$ of the participants are impacted by digitalization. About $59 \%$ of the involved participants were considered very strong, $35 \%$ were strong and $6 \%$ were considered low. These findings show that information technology and IPA play a significant role in industries. This can be considered by the findings that indicate the state of digitalization the surveyed participants were in. About $38 \%$ were in their development stage, $51 \%$ at their stage of transition and about $11 \%$ at their commencing stage of IPA.

Approximately $39 \%$ of the respondents operated in IT industries, $19 \%$ were in professional service sectors, $18 \%$ in retailing stores, $15 \%$ in logistic companies and $9 \%$ in financial institutions. The chosen industries can be classified as $52 \%$ in middle class and family business classes, $27 \%$ in internet business and start-up businesses and $21 \%$ in enterprise businesses. About $51 \%$ of the surveyed participants' rated information was company data, e.g. process data, optimization data and strategic assets. About $39 \%$ of the participants evaluate data as privacy issues and feasible data and about $9 \%$ of the participants are skeptical and visualize information as drivers of costs.

About $100 \%$ of the participants were aware of, utilized business process management, and automated applications for business process management. About $12 \%$ utilizes business intelligence models, data warehouses and data lakes for data management and data reporting. About $75 \%$ of the participants are aware of AI and ML, but about $29 \%$ actually evaluate ML. About $26 \%$ are aware of deep learning and just $2 \%$ execute it. The usage of ML and AI at the present and in the future is indicated in the Fig 3 below.

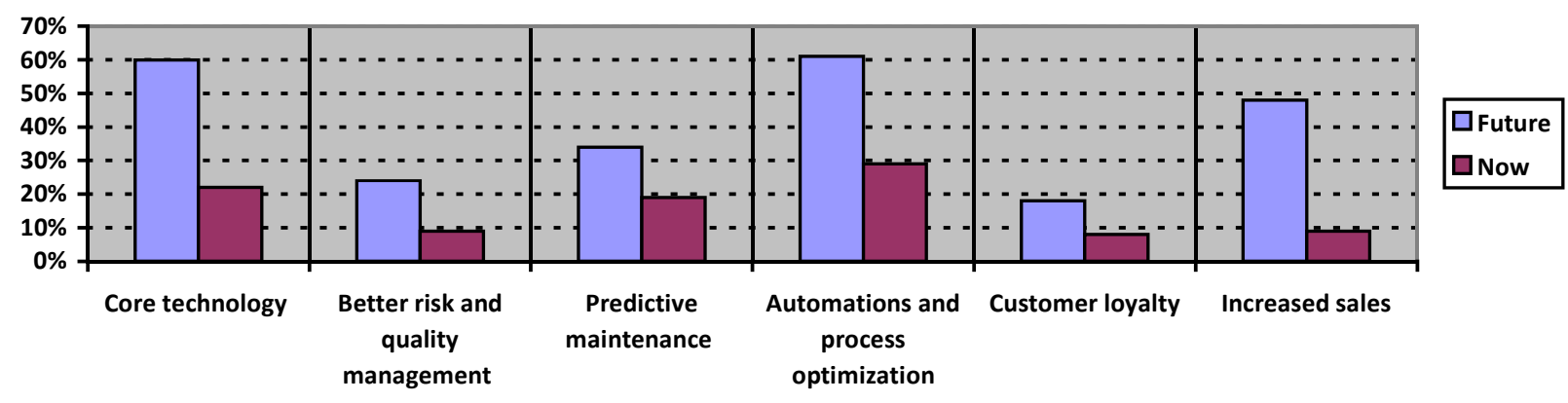

Fig 3: Application of AI and ML now and in the future

It can be visualized that the optimization of processes with $29 \%$ of the participants is the most fundmaental and evaluated application in the survey. Othe applications such as ML, as a ccritical technology for novel business frameworks or predictive maintainability are the second and third significant segments of the application. The estimation and outlook to the year 2021 indicates the affirmed position of processes requiring more automation and process automation. In addition, the enhanced value of applications in critical technologies indicate the implication of continued digitalization. The major phase in the field of ML can be considered in sales.

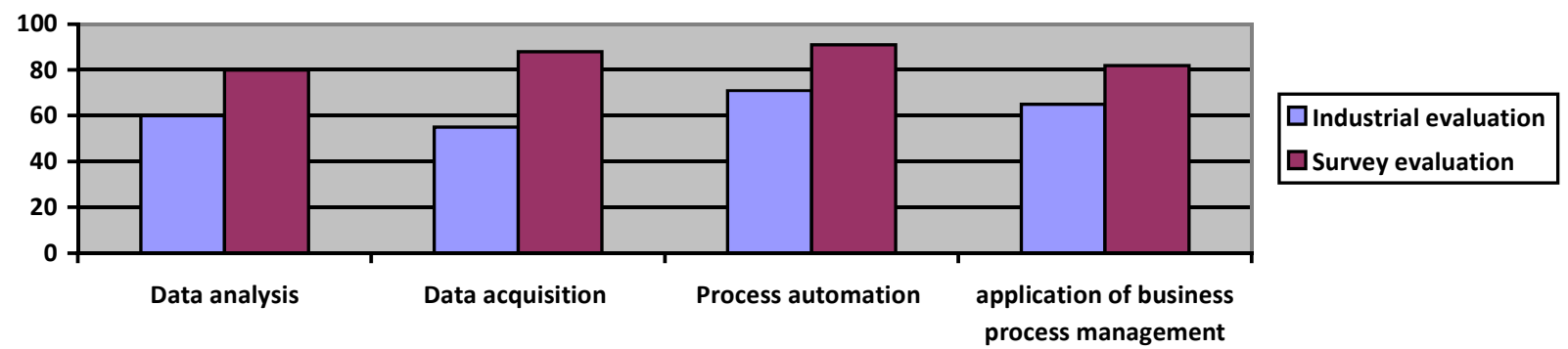

Fig 4: Shadowing vs interview findings 
The results of shadowing vary between individual assessment of the survey participants. As a benchmark, the elevated infromation of the interviews and surveys will be set. According to Fig 4, many participants asset their application of business process management as up-to-date and process controlling and automation as effectively set up.

The survey evaluation projects $87 \%$ of all the queries in Fig 4 . The compared value during shadowing is about $15 \%$ less by $62 \%$. This indicates the varied exceptions and significant value for the development. Certainly, process automation points indicate a varied $20 \%$ in business and theory. The gaps can cause a major issue e.g. missed business process management and missed continual advancement. To deal out these business problems, halfautomated processes and resource wastes, baseline data concerning particular fields e.g. processes, have to be set afresh by industries. In connection with comprehensive data analysis, ML and deep learning, the automation and optimization can be identified.

\section{DISCUSSION}

Tradition manufacturing business models are constantly changing and novel models are emerging; incumbents have to be quick at recognizing and reacting to competitive challenges. Stimulated by the internet, virtual and real worlds are advancing ever further from IoT and Cloud Computing. Cloud Computing is hosted on the internet and permits remote accessibility to services and apps to manage machines, workflows and processes. IoT builds on this concept by utilizing cloud system to automate and store in objects, which are synchronized to the internet e.g. remote home lighting, shade systems and internet-enabled automobiles.

Industry 4.0 considers the management of processes into the cloud system where they are easily managed from anywhere. In most cases of Big Data, rapidly transforming conditions in process management, process automations, customer requirements and supply chain play a critical role in minimizing the response time to eliminate back logs, enhancing the processing of errors, and dissatisfied customers. To facilitate a further step in process automation and management, this paper look further into the possibility of applying AI and ML for IPA and management in IPA. The opportunities of independent management of processes, through learning and automated computing programs promise in automations, flexibility and theory agility for industries and relevant stakeholders.

From this research, about $80 \%$ of the survey participants concerning ML, AI and deep learning as synonyms that imply to the same concept. According to the description in the theoretical segment, ML is considered as a subsegment of AI. The findings of the survey indicate the present demand for AI and ML for automation and optimization of process and support of business process management. In addition, the participants assess the applications of ML as very essential for the future of industries. Not just for the optimization of processes, but all as a core competency for novel business models and for enhanced sales support.

The findings of shadowing indicate two major results: The first one is that the participants estimate their usage of business process management and automatic process management more effective compared to reality. The second one is that about $45 \%$ of the compiled information from industries are not controlled or utilized in an effective manner. This indicates a major space and opportunity for advancement only in the transformation of process management. Data lakes and big data are utilized in the business; however, the analysis and reporting of data can be developed. ML, $\mathrm{AI}$ and deep learning can be considered as a recommendation to optimize and automate the entire process and support business process management via novel algorithms and independent learning through constant evaluation of data.

\section{Theoretical Assumptions}

\section{1) Traditional Concepts and Machines}

The ideology of artificial intelligence is not as innovative as it might tend to be. Actually, some individuals argue that the ideology of AI was considered from the Ancient Greeks. Nonetheless, without touchable computers and machines, humans cannot have established any forms of mechanical automations in industries. In addition, the history of IPA begins far-off near than Socrates and Aristotle. The traditional aspect of automation begins with industrial revolution and equipment of industries between 1790 and 1840. Before, just like now, industries dreaded the impact of IPA on their jobs (however, this turned out well in the end of it). In 1837, Charles Babbage began the establishment of simple machines known as Analytical Engines that were considered the first devices to be called computers. Ada Lovelace developed the first programs for computers that would have operated on machines. However, Babbage expired before the prototype was finished.

It is significant to evaluate why IPA is the required of the present. With massive data and enhanced automation competency, business administrations are searching on how they can advance business procedures to attain significant operational productivity. They are probable to identify that the machines operate effectively until shapeless sets of data are considered in the mix. At that moment, the process starts or stops in the pathways. This is an issue of industrial development, which takes full advantage of what robotic process automations have to provide. In addition, it involves the integration of great efficacy and cost of ownership for automation creativities.

Documents and amorphous information, e.g. websites, communication, photographs, videos and PDF, make complete end-to-end automations of commercial operation challenging since they necessitate humans to assess, comprehend and make necessary conclusions based on data checked. This establishes blocks and intensely indicates workflows, which are an opposite of what industries purpose to accomplish with their automation creativity. This condition is far from rare, making it a critical risk to IPA ambition. As much as $60 \%$ of industrial process integrates 
some types of formless data. This implies that $60 \%$ of the time robots have to halt their work ups until the mediation of humans. For instance, in the entitlement-processing segment, approximately every characteristic of process remainders are paper-based.

Individuals email and main scanned or physical documents in systems, where humans have to organize and review them by hand. For individuals with complete automation ambitions, this is challenging. It also can clarify why, irrespective of two periods of applications for business process management, complete process automation is still exclusion. About $2 / 3$ of industries are less than $49 \%$ mechanical. In addition, whereas some entrepreneurs are utilizing Robotic Process Automations (RPA) to record customer communication, record management activities, evaluate processing activities and paper-exhaustive procedures, less than 5 companies have completely automated most of their central back end processes.

It was noted that the problem due to formless data would be inferior. Half of the survey participants comment that $60 \%$ of data in their company is formless. At the same moment, companies are antedating massive data development. With respect to the survey, $35 \%$ project the amount of data to enhance quintuple over the two next years. About $69 \%$ of companies surveyed comment that unstructured data is 'Achilles Heel' for various employments of RPA. To

attain stable automation levels, industries will require connecting RPA with AI, a core capacity of a smart automation phase. With enhanced reasoning detection and firm examining, removal and interpret formless information becomes authenticity.

Intelligent automations allow companies to digitally transform knowledge-based industrial processes hence transforming their ambitions into satisfying dreams. A platform of intelligent automation can potentially manage document parting, directing, and organization, and enhancing the speed of accuracy and processing, whereas minimizing the need to human involvement. Therefore, tasks, which initially derailed robots, will be handled in a proficient manner. Relate what happens when a client annoying to open an account through a bank's request uploads photos of a driver's license. The photos have to be read and information removed, or how RPA deals with patients' email, which integrates fundamental details about recent entitlements.

In both instance, RPA bots cannot deal with the urbane information. Humans have to step in to deliver, comprehend and draw essential conclusions. However, an intelligent automation platform organizes this and more. With the aid of intellectual document automations, the platform imprisonment reads and comprehends the data. Because intelligent document automation can read information in viability of arrangement, it can change the driver's certificate and emails into usable data. Using ML and natural language, platforms of intelligent automation then comprehends the data and governs what takes place next.

Intelligent automation platforms grip this task more effectively and at a pocket-friendly price compared to bolt on remedies. This allows industries to form significant competences minimal TCO, and completely automate industrial processes endwise. For industries that are stressed to attain developed automation levels because of data limitations forming slowdowns and blockages, a critical thought has to be applying solutions, which integrate RPA with AI. Instead of enduring the situation, industries can enhance automaton advancements from boring transactional cases to critically multi-faceted skill-base industrial procedures, ornamental client experience and operative excellence. With the involvement of intelligent automation and AI skills, industries can start operating and attain significant statures in automations.

\section{Variation between AI and Automations}

RPA software is significant for undertaking simple tasks, which follow systems and instructions set by industries. RPA has the capacity to execute various tasks with high degree accuracy that humans cannot complete. It is effectively suited for significantly foreseeable and repetitive tasks. Automated tools necessitate guide outlines and the management of humans to effectively underpin campaigns. RPA tricks are for humans to antedate each variation so that machines can automatically operate in the right manner each time. This explains why constant attentiveness is essential. In case the ecosystem changes, industrial marketers must manually be engaged in the process to make critical changes.

AI reflects on the manner in which computer models can utilize massive data to copy human intelligence, understanding systems and enhance the learning, prediction and commenting on forthcoming events. AI that is able to sympathetically market KPIs can utilize different algorithms, which act in performances to identify the signals in data noise and identify the tracks to remedies, which no humans can attain. Wide-range AI presently operate in an assistive manner in case the next best action is referred to human who can therefore decide if they can rely on them or not and therefore make physical transitions. Differences between automation and AI have been provided in Table 1.

Table 1: Key differences between $\mathrm{AI}$ and automations

\begin{tabular}{ccl}
\hline No. & \multicolumn{1}{c}{ AI } & \multicolumn{1}{c}{ Automations } \\
\hline $\mathbf{1}$ & $\begin{array}{l}\text { AI executes decisions with respect on } \\
\text { learning from previous experiences } \\
\text { and data it accepts }\end{array}$ & $\begin{array}{l}\text { Automations are like the presets and } \\
\text { individual operations meant to perform } \\
\text { particular obligations }\end{array}$ \\
& $\begin{array}{l}\text { AI represents a system, which aids } \\
\text { professionals to evaluate remedies and } \\
\text { reach standardized conclusions }\end{array}$ & $\begin{array}{l}\text { Automations are a form of machines } \\
\text { that have been created to complete a } \\
\text { particular routine task }\end{array}$ \\
\hline $\mathbf{3}$ & AI represents non-repetitive & Automations denote the repetitive \\
\hline
\end{tabular}




\begin{tabular}{|c|c|c|}
\hline & obligations & $\begin{array}{l}\text { obliigations with respect to rules and } \\
\text { commands }\end{array}$ \\
\hline 4 & $\begin{array}{l}\text { AI incorporate the interactions of } \\
\text { humans and masters from previous } \\
\text { experiences and makes a comparison } \\
\text { of the situations annd thereby operates } \\
\text { according to these situations }\end{array}$ & $\begin{array}{l}\text { Automations do not have interactions } \\
\text { with humans and hence operate under } \\
\text { no instructions }\end{array}$ \\
\hline
\end{tabular}

\section{Combination of AI and $M L$}

Whenever robotic process automations are incorporated with elements of ML and AI, the findings are considered Intelligent Process Automations (IPA). APA tools are considered effective since it consents us to earn the merits of automation, enhance speed, saves time, enhance efficiency and enhance the capacity to measure with processing energy, elasticity and vision of AI. Sellers utilizing IPA can advance their skills, whereas off-loading operational management tasks that are boring for machines. This is different from pure robotic automation where AI can begin, halt or transform what is doing with respect to the atmosphere in which the machine operates.

In addition, since the best systems of AI permit selects to establish railings, there are no chances of unexpected events considering findings too far astray. For sellers, this implies sooner, more modified processes and implementation, significant correctness and use in data and advancements in general experience of the customer. Industrial marketers transform from bothering over budget distribution and bid modification to higher value-added human-based donations such as the development of value intention to develop business process. Due to clear profits, Forrester projects that by end of $2021,25 \%$ of the companies (fortune 500) will report many examples of IPA. Certainly up to 2050, industries will depend on AI and automations since it will make life more accurate and easier.

\section{Advantages of AI and ML for Industrial Marketers}

IPA initiates are not just providing exterior visions for industrial marketers but also transform visions in actions. For instance, Albert can potentially produce historical digital operational information across multiple channels, explore different message groupings, construct implementation policies, and enhance creativity and project occurrences. Developing unremittingly over significant time, intelligence autonomous competency of machines permits it to really transform finances, change offers and optimize operations that an industrial marketer can set. This is fundamental since the customer can continue to demand more products.

IPA initiatives are presently becoming the best way to distribute modified tracing points for optimum client experience over salaried digitalized channels. This discussion indicates excessive application and demand for automations and AI advances on a daily basis. Probably until 2050, we will fully rely on AI and ML since it makes life more accurate and easier. Table 2 below shows the probability of ML in various fields.

Table 2: Probabilities of ML in various fields

\begin{aligned} & \hline ML probabilities (\%) \multicolumn{1}{c}{ Application } \\ & \hline 97 General secretarial tasks \\ & 97 Bank counter staffs and advisers \\ & 96 Mobile phone operators \\ & $\mathbf{9 0}$ Cashiers \\ & $\mathbf{8 5}$ Post-distribution auditors \\ & $\mathbf{8 2}$ Printing \\ & $\mathbf{7 2}$ Agricultural workers \\ & \hline\end{aligned}

\section{Components of AI in Automation}

Intelligent ML systems purpose to utilize three fundamental mechanisms of AI. Based on the present need, they can be used collectively or utilize to distinctly formulate a completely automated remedy.

\section{Machine Visions}

The vision of machines represents the potential of programs to identify photographic inputs. Machines utilize drill information or images as a basis for the industry and to potentially document mechanisms. A suitable case of machine visions is face recognition used in mobile phones such as iPhone. Face recognition is an effective modern future in iPhones. AI investigate database using by Facebook is the best example of investigative image, which explains, organizes, quantifies and classifies novel illustrations to users.

Natural Language Processing (NLP)

Where machine vision operates on an illustration, NLP does the same thing to identify the voice of humans and text input. We come a long way in forming NLP. Presently, it is comprehensive for machines to structure the context 
following the announcement and consider following actions with respect to prebuilt background and data variables. Google Assistance, Amazon Alexa and Apple's Siri all depend on NLP to present value to workers.

Machine Learning (ML)

ML is the capability of machines to acquire from the information fed, results of conclusions and variables of the atmosphere to enhance itself. Using ML, we can enhance effectiveness of the present resolutions. For instance, if a problem arises under intelligent automation systems and human engagement, the next step is to solve it before engaging the mechanical input of humans. Therefore, over time, competence will develop and human efforts will degrade. Feasibly until 2050, we will fully rely on AI and automations since it makes life more accurate and easier.

\section{Why Select Intelligent Process Automation (IPA)}

The application of AI and ML in automation makes it easier for industries to complete routine operation [21]. Therefore, here are details why applying IPA is advantageous.

\section{Cost Reduction}

The rate of charges of training humans in task of routine nature is its periodic nature. You will have to deal with business revenues, provide time for the expansion of skills and undergo occupational charges. Contrary to that, machines and robotic systems once effective only recover and has no cost intricate in repetitive training [22].

\section{Enhanced Efficiency}

Irrespective of how well organized the workforce might be, people are fond of making mistakes. In that case, an automated remedy (machine) is infallible and is considered to make little to no mistakes. As time goes, it can absorb from the outputs; thereby, improving its efficiency.

\section{Novel human obligations}

Like computers, AI will also transport a completely novel human obligation. Individuals with extraordinary competencies will find themselves exercise low-level automations systems to do most of the work.

Selecting the best process to implement IPA in industries can be challenging. For some companies, the first issue is where to implement IPA. Even though IPA provides wide-range of benefits, various industries and their workers are used to set systems and developments. In addition, over-transforming towards automations can be both costintensive and devastating for workers and the company. For instance, if labor-intensive processes are affordable for the industry in contrast for process automation, the firm will spend automations for development since large-scale savings will be involved to generate satisfactory return. Therefore, choosing the risk process and right time for IPA is the present issue for industrial development in the competitive market. Fig 5 represents IPA size from $2016-2022$.

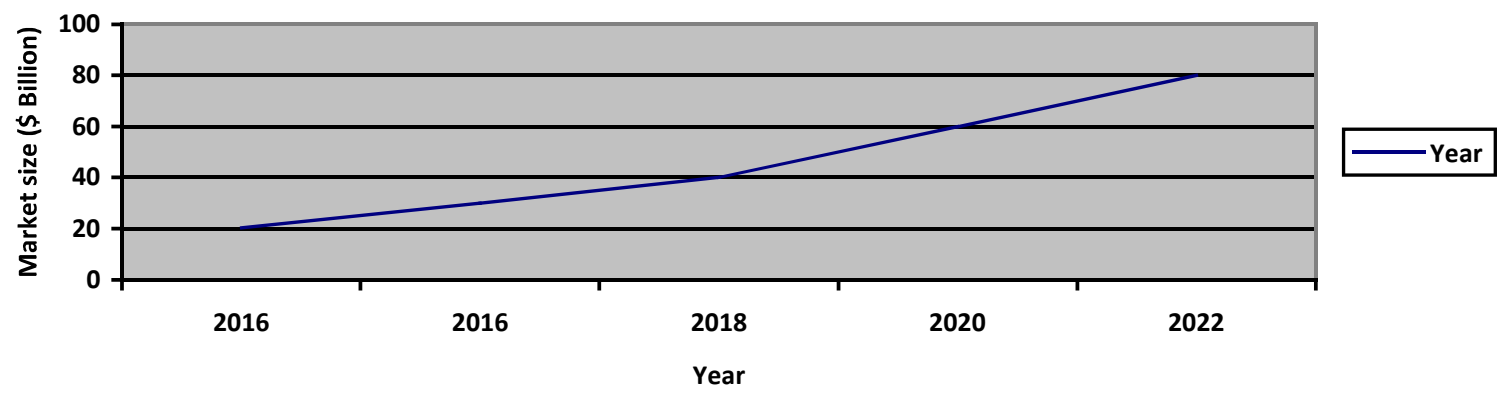

Fig 5: IPA market size from 2016 to 2023

\section{CONCLUSION}

In conclusion, ML and AI is a product of scientific development. The idea in this paper is that that machinery could perform and contemplate tasks just like humans. Digitalization cannot be stopped and changes in the industrial market situation in many companies are presently evident. Time-intensive workflows, based on excel, paper work, and email minimize the productivity rate of industries. In this paper, it can be argued that everything that can be automated will be automated. Every process, business model and product becomes automated; in that case, revolution depends on the processes. Optimized process management is possible through digitalization and integration of automatic processes is effectively supported by ML and AI. In that case, consistent and targeted process automations are a critical factor of success presently and in the future according to survey findings. It is increasingly fundamental for industries to apply power of datasets to make profitable and smart decisions. 


\section{References}

[1]. R. Oakey, "Are disruptive technologies disruptive [disruptive technologies]", Engineering Management, vol. 17, no. 2, pp. 10-13, 2007. Available: 10.1049/em:20070201

[2]. S. Robbins, "AI and the path to envelopment: knowledge as a first step towards the responsible regulation and use of AI-powered machines", AI \& SOCIETY, vol. 35, no. 2, pp. 391-400, 2019. Available: 10.1007/s00146-019-00891-1.

[3]. I. Parshina and E. Frolov, "Development of a digital twin of the production system on the basis of modern digital technologies", Russian Journal of Industrial Economics, vol. 13, no. 1, pp. 29-34, 2020. Available: 10.17073/2072-1633-2020-1-29-34.

[4]. A. Ogawa and T. Hori, "Error detection and accuracy estimation in automatic speech recognition using deep bidirectional recurrent neural networks", Speech Communication, vol. 89,pp. 70-83, 2017. Available: 10.1016/j.specom.2017.02.009.

[5]. H. Miyazaki, "Information Technology in Industrial Applications. III. AI, Petri Net, \& Genetic Algorithm Application Technology in Industrial Systems. 1. AI Application Technology.", IEEJ Transactions on Industry Applications, vol. 113, no. 12, pp. 1355-1358, 1993. Available: 10.1541 ieejias.113.1355.

[6]. K. Tangrand and B. Bremdal, "Using Deep Learning Methods to Monitor Non-Observable States in a Building", Proceedings of the Northern Lights Deep Learning Workshop, vol. 1, p. 6, 2020. Available: 10.7557/18.5159.

[7]. O. Prokopenko and V. Omelyanenko, "Marketing aspect of the innovation communications development", Innovative Marketing, vol. 14, no. 2, pp. 41-49, 2018. Available: 10.21511/im.14(2).2018.05.

[8]. S. Kwak, "Strategies on Overseas Direct Sales in Cross-Border B2C e-Commerce", The e-Business Studies, vol. 18, no. 6, pp. 279-296, 2017. Available: 10.20462/tebs.2017.12.18.6.279.

[9]. I. Lee and Y. Shin, "Machine learning for enterprises: Applications, algorithm selection, and challenges", Business Horizons, vol. 63, no. 2, pp. 157-170, 2020. Available: 10.1016/j.bushor.2019.10.005.

[10]. H. Yang, "A special issue on: Bayesian statistics and machine learning in business", Applied Stochastic Models in Business and Industry, vol. 32, no. 3, pp. 309-310, 2016. Available: 10.1002/asmb.2172.

[11]. P. Addo, D. Guegan and B. Hassani, "Credit Risk Analysis Using Machine and Deep Learning Models", Risks, vol. 6, no. 2, p. 38, 2018. Available: 10.3390/risks6020038

[12]. M. Leo, S. Sharma and K. Maddulety, "Machine Learning in Banking Risk Management: A Literature Review", Risks, vol. 7, no. 1, p. 29, 2019. Available: $10.3390 /$ risks7010029.

[13]. G. Keilbar and W. Wang, "Modelling Systemic Risk Using Neural Network Quantile Regression", SSRN Electronic Journal, 2020. Available: $10.2139 / \mathrm{ssrn} .3685748$.

[14]. S. PK, "Enhancing User Experience Using Machine Learning", International journal of simulation: systems, science \& technology, 2019. Available: 10.5013 /ijssst.a.20.05.01.

[15]. T. Wuest, D. Weimer, C. Irgens and K. Thoben, "Machine learning in manufacturing: advantages, challenges, and applications", Production \& Manufacturing Research, vol. 4, no. 1, pp. 23-45, 2016. Available: 10.1080/21693277.2016.1192517.

[16]. S. Konopatov and N. Saliyenko, "Platform-based business models", Economics and Environmental Management, pp. 21-32, 2018. Available: 10.17586/2310-1172-2018-11-1-21-32.

[17]. P. Pereira, "Using ML Models to Detect Malicious Traffic: Testing ML Models", International Journal for Information Security Research, vol. 10, no. 1, pp. 906-909, 2020. Available: 10.20533/ijisr.2042.4639.2020.0104

[18]. A. Rogers, O. Kovaleva, M. Downey and A. Rumshisky, "Getting Closer to AI Complete Question Answering: A Set of Prerequisite Real Tasks", Proceedings of the AAAI Conference on Artificial Intelligence, vol. 34, no. 05, pp. 8722-8731, 2020. Available: 10.1609/aaai.v34i05.6398.

[19]. V. Reshetnyk, "Information in the Natural Cyber Systems (To Definition of the Term Information)", Journal of Automation and Information Sciences, vol. 52, no. 8, pp. 59-67, 2020. Available: 10.1615/jautomatinfscien.v52.i8.50.

[20]. S. Dey and A. Das, "Robotic process automation: assessment of the technology for transformation of business processes", International Journal of Business Process Integration and Management, vol. 9, no. 3, p. 220, 2019. Available: 10.1504/ijbpim.2019.100927.

[21]. K. Gharehbaghi, "Process Automation in Intelligent Transportation System (ITS)", International Journal of Machine Learning and Computing, vol. 8, no. 3, pp. 294-297, 2018. Available: 10.18178/ijmlc.2018.8.3.702.

[22]. V. Kommera, "Robotic Process Automation", American Journal of Intelligent Systems, vol. 9, no. 2, pp. 49-53, 2019. Available: 10.5923/j.ajis.20190902.01 